University of Nebraska - Lincoln

DigitalCommons@University of Nebraska - Lincoln

USDA Wildlife Services - Staff Publications

U.S. Department of Agriculture: Animal and

Plant Health Inspection Service

2009

\title{
Options for the Control of Disease 3: Targeting the Environment
}

\author{
Alastair Ward \\ Kurt C. Vercauteren \\ USDA-APHIS-Wildlife Services, kurt.c.vercauteren@usda.gov \\ W. David Walter \\ Emmanuellle Gilot-Fromont \\ Sophie Rossi
}

See next page for additional authors

Follow this and additional works at: https://digitalcommons.unl.edu/icwdm_usdanwrc

Part of the Environmental Sciences Commons

Ward, Alastair; Vercauteren, Kurt C.; Walter, W. David; Gilot-Fromont, Emmanuellle; Rossi, Sophie; EdwardsJones, Gareth; Lambert, Mark S.; Hutchings, Michael R.; and Delahay, Richard J., "Options for the Control of Disease 3: Targeting the Environment" (2009). USDA Wildlife Services - Staff Publications. 984.

https://digitalcommons.unl.edu/icwdm_usdanwrc/984

This Article is brought to you for free and open access by the U.S. Department of Agriculture: Animal and Plant Health Inspection Service at DigitalCommons@University of Nebraska - Lincoln. It has been accepted for inclusion in USDA Wildlife Services - Staff Publications by an authorized administrator of DigitalCommons@University of Nebraska - Lincoln. 


\section{Authors}

Alastair Ward, Kurt C. Vercauteren, W. David Walter, Emmanuellle Gilot-Fromont, Sophie Rossi, Gareth Edwards- Jones, Mark S. Lambert, Michael R. Hutchings, and Richard J. Delahay 


\title{
Chapter 8 \\ Options for the Control of Disease 3: Targeting the Environment
}

\author{
Alastair I. Ward, Kurt C. VerCauteren, W. David Walter, \\ Emmanuelle Gilot-Fromont, Sophie Rossi, Gareth Edwards-Jones, \\ Mark S. Lambert, Michael R. Hutchings, and Richard J. Delahay
}

\subsection{Introduction}

Management of wildlife disease can be targeted at pathogens, hosts or vector populations, but may also focus on the environment. As constituent elements of any given environment, resident wildlife populations, and their pathogens, may be profoundly influenced by environmental change, in terms of their abundance, distribution and behaviour. Hence, it is reasonable to expect that incorporation of environimental manipulation into a programme to control wildlife diseases may potentially result in outcomes as effective as direct intervention aimed at hosts, pathogens and vectors.

Environments are not static, but are naturally dynamic, complex systems that exert strong influences on patterns of disease via their impact on hosts, pathogens, vectors and the interactions between them. Consequently, it can be difficult to identify which environmental variables are most important in influencing disease dynamics and hence which elements to target as part of a disease management programme. Nevertheless, environmental management has been used extensively to control

A.I. Ward, M.S. Lambert, and R.J. Delahay

Central Science Laboratory, Sand Hutton, York, YO4 I ILZ, UK

K.C. VerCauteren and W.D. Walter

USDA, APHIS, Wildlife Services, National Wildlife Research Center, Fort Collins, CO, USA

E. Gilot-Fromonc

Université de Lyon; Université Lyon 1; CNRS; UMR5558, Laboratoire de Biométrie et Biologie Evolutive, 43 Boulevard du $1 /$ Novembre 1918, Villeurbanne F-69622, France

S. Rossi

Unité Sanitaire de la Faune, Micropolis, la Bérardie, Belle Aureille, 05000) GA.P, France

G. Edwards-Jones

School of the Environment and Natural Resources, University of Wales, Bangor, Gwynedd, LL57 2UW. UK

M.R. Hutchings

SAC. West Mains Road, Edinburgh, EH9 3.JG, UK 
diseases in wildlife in many parts of the world, with some apparent success (Wobeser 2002). Anecdotal information arising from disease management projects and from studies of wildife behavioural ecology and disease epidemiology suggests that environmental manipulation may offer potential opportunities for the long-term management of many diseases of wildlife. However, while more direct approaches to disease management, such as host population reduction (see Chapter 7) or vaccination (see Chapter 6), might have rapid effects, the benefits of environmental manipulation are likely to take much longer to accrue.

In this chapter we investigate relationships between wild mammals, their environment and disease dynamics. We then discuss the potential applications of environmental management as a tool for managing wildlife diseases, with reference to case studies.

\subsubsection{The Environment-A Definition}

The environment may be described in its widest sense as the conditions in which an organism lives, including the influences of all biotic and abiotic components. The topography of the physical environment is heavily influenced by the underlying geology, which influences the distribution of soils, vegetation and surface water. Superimposed onto this natural landscape are all the artefacts of human infrastructure. The vegetation communities that cover the land surface are a particularly important component of landscape structure in terms of mammal distribution. Their diversity provides a wide range of niches for mammals to inhabit. Even within a given vegetation community, structure varies, with canopy, sub-canopy and ground-level species contributing to the character of landscapes and influencing ecological processes. In this chapter we acknowledge this complexity and define the environment as the land, water bodies, natural and man-made structures, substrates and vegetation within which wildlife and their associated pathogens exist.

\subsection{Environmental Management}

Humans are prodigious engineers of their environments, pursuing management in the interests of agriculture, urbanisation and infrastructure development, and to enhance wildlife populations for food, leisure and (at our most enlightened) to conserve biodiversity. Environmental management has also been used historically to manage wildlife diseases. Such strategies have usually targeted host contact with pathogens, for example by using fencing to prevent wild mammals from gaining access to water holes infected with Bacillus anthracis (the causative agent of anthrax) (Hugh-Jones and de Vos 2002) and vector control, such as prescribed burning of forest.vegetation to reduce tick populations (Allan 2001). 
Few controlled experiments have been undertaken to determine the effects of environmental manipulation on wildlife disease dynamics or the distribution and abundance of pathogens of wild manmals. One exception was an experimental application of herbicides and vegetation burning to alter plant communities, which also affected the distribution, species richness, abundance and prevalence of helminths in their cotton rat (Sigmoidon hispiclus) hosts (Boggs et al. 1991). It was suspected that vegetation management had altered local microclimates, thus affecting the survival of free-living stages of the helminth parasites. This study clearly illustrates the potential for environmental management to be used to target pathogens. An alternative approach would be to control pathogens by targeting environmental manipulations at their hosts or vectors, although reports of such experimental studies are rare. Nevertheless, countless ecological studies have described how wild mammal populations respond to environmental changes by altering their patterns of space use (see Box 8.1). For example, changing agricultural practices can lead to removal of food resources and cover for roe deer (Capreolus capreolus) causing them to shift their home ranges and alter their habitat use and spacing patterns (Cimino and Lovari 2003). Interpretation of these effects in the context of disease management suggests that alteration of habitat composition and structure could hold potential for manipulating local host densities and contact rates, with direct consequences for the transmission of infectious diseases.

\section{Box 8.1 Uabitat management and rat control}

Rats are pertiaps the most motorious of all mammaliandisease vectors. Their his torical association with the bubonic plague still endures, even though the ship rats (Rattus rattus) that carried plague (Yersinia pestis) throughout Medieval Europe thave long since been replaced by the Norway rat (Rattus nonvegicus) in most temperate regions Norway rats rarely carry the Oriental rat flea (Xenopsylla cheopis:), usually responsible for transmission of the plague bacteria from infected rodents to other animals, although they have been identified as reservoirs and vectors of many other zoonoses. Norway rats collected from UK farms were found to be carrying 13 zoonotic and 10 non-zoonotic parasites, including Cryptosporidium, Pasteurella, Listeria, Yersinia, Coxiella and Hantavirus (Webster and Macdonald 1995). Norway rats have also been suggested as potential vectors of footand:mouth disease in the UK (Capel-Edwards 1970), as they are highty mobile and could therefore carry infective material between farms.

Most disease transmission from Norway rats to livestock probably occurs indirectly, throughi contamination of food sources or incidental contact with rat urine and faeces. Rodent proofing of buildings can be an'effective way of reducing. direct and indirect contact betweein rats and livestock, but may not always be practical, especially on older buildings. Another option is to teduce rat populations using rodenticides. This can be effective in the shott term, but rat populations have 
Box 8.1 (continued)

a considerable capacity for recovery through compensatory reproduction, and hence repeated applications of rodenticide sometimes become necessary. However, this incurs a serious risk that rodenticide resistance will develop (Cowan et al. 1995). The need for repeated lethal control could be reduced if attention were given to the reasons why rat populations become established, and if means could be identified of modifying the environment to make it less attractive to rats. The removal of scrub vegetation adjacent to Australian macadamia orchards helped control rat damage (White et al. 1998) and clearing refuse and overgrown areas reduced the size of rat populations in urban areas of the USA (Jackson 1998) and on UK farms (Lambert et al. 2008). Of course it is not possible to remove all areas of harbourage, so periodic and well-targeted rodenticide treatments may still be necessary. Reducing rat immigration from surrounding areas may decrease the need for rodenticides still further. Studies of radio-tagged rats suggest that they tend to avoid open areas, and probably move between farms using hedgerows and ditches as cover. The extent to which immigration contributes to the recovery of rat populations following rodenticide treatments is unclear, and in the UK it is unlikely that large-scale migrations across farmland occur. Even so, targeted trapping of rats along field margins and hedgerows might be usefil in reducing the potential for disease transmission between farms.

\subsubsection{Effects of Environmental Management on Disease}

Naturally occurring host-parasite systems may evolve over time to reach a relatively stable equilibrium. However, dramatic changes, such as might be caused by human activities, can disrupt this endemic stability and result in disease outbreaks. The loss, degradation and fragmentation of wildlife habitats, largely through human encroachment, are not only responsible for substantial reductions in biodiversity but are also considered to be major causes of disease outbreaks in some mammals (McCallum and Dobson 2002).

Habitat fragmentation can result from expanding agriculture, silviculture or urbanisation and can lead to a reduction in available habitat for wildlife, thus altering space use and contact rates between wild and domestic animals and humans, with implications for the transmission of pathogens. African wild dog (Lycaon pictus) populations for example, have decreased in size in parallel with human population growth. While habitat loss and fragmentation, and increased persecution owing to human population expansion are considered to be the main causes of wild dog population declines, disease has been a significant source of mortality, particularly during episodic outbreaks (Woodroffe and Ginsberg 1999). Domestic dogs (Canis lupus familiaris) have probably been the predominant source of infection, and the likelihood of their contact with wild dogs has increased as human populations have expanded towards protected areas. 
Human activities may degrade habitats in a variety of ways, including physical alteration, simplification of habitat structure and pollution. Some pollutants including heavy metals and polychlorinated biphenyls (PCBs), can directly compromise mammalian immune systems and thereby increase susceptibility to disease (Exon et al. 1985; Hilliam and Ozkan 1986).

Increased habitat fragmentation was predicted to result in the extinction of Chlamydia psittaci (a sexually transmitted infection) from wild koala (Phascolarctos cinerus) populations (Augustine 1998), which may, at face value, seem like a good thing. However, habitat fragmentation was also predicted to enhance the risk of extinction of koalas caused by infection with the parasite. In undisturbed environments koalas and Chlanyclia co-exist within a natural, stable host-parasite relationship, and so it has been argued that loss of the parasite from this system would diminish native biodiversity (Augustine 1998).

Clearly, land management can have a considerable impact on diseases in wild mammal populations. The increasing global use of environmental impact assessments (EIAs) during development projects, offers a potential methodological framework in which to address and perhaps mitigate detrimental effects on disease dynamics. However, EIAs and risk assessments incorporating the effects on diseases of wildlife are far less common than those involving diseases of humans and livestock. An example of the latter is provided by an assessment of the impacts on human health of surface and sprinkler crop irrigation systems in Zimbabwe (Chimbari et al. 2004). The authors compared records of malaria and schistosomiasis from health centres serving areas with either type of irrigation scheme, and a location where no irrigation occurred. Their parallel risk assessment approach suggested that poor land management (e.g. inadequate drainage and accumulation of surface water) and poor maintenance of sprinkler equipment were most likely to be responsible for variations in disease incidence because they created suitable breeding habitat for mosquito vectors and sniail hosts. Similar risk assessment methods could be used to assess the impacts of land development on diseases in wildlife. The limited use of this approach to date probably reflects our relatively poor understanding of the implications of changes in land management for wildlife disease dynamics.

\subsection{The Importance of Landscape Structure}

Landscape structure influences networks of host-pathogen contacts and thus the dynamics of diseases in wild populations. Models of disease in metapopulations (i.e. discrete but.inter-connected patches of sub-populations of organisms) predict that spatial heterogeneity increases disease persistence (Post et al. 1983; Wood and Thomas 1996), drives epidemic cycles (Bolker and Grenfell 1995) and influences the evolution of parasite virulence through local adaptation (Lively 1989). These processes have yet to be demonstrated for wild mammals but the influence of spatial heterogeneity on pathogen transmission among invertebrates is well documented. For example, parasite transmission amongst barnacles (Chthamalus 
dalli) was enhanced by increasing both host density and the heterogeneity of their distribution (Blowier and Roughgarden 1989).

The inclusion of landscape structure in disease management plans requires the availability of data on its influence on disease dynamics, ideally from experimental studies where cause and effect can be demonstrated. In practice however, correlative data may be all that are available and putative landscape effects may have to be cautiously inferred. Spatial modelling using geographical information systems (GIS) can be used to simulate the complexity of landscape structure and to investigate interactions with hosts and pathogens. Landscape data may be used to predict the environmental carrying capacity of a host population, contact patterns (diffusion) and the persistence of a pathogen in the environment. For vector-borne diseases such as malaria and West Nile virus, risks of disease spread may be predicted by mapping the distribution of habitat favpurable to vectors. For example, remotelysensed data within a GIS was used to monitor changes in artificiäl aquatic habitats in Wyoming, USA (Zou et al. 2006). This identified favourable sites for the development of larval mosquitoes, which may carry West Nile virus. Monitoring the location of such habitats could be used to predict vector distributions, and so help to more effectively target control efforts.

Landscape structure may also influence the efficacy of disease management measures where the terrain imposes limitations on the practical implementation of field operations. For example, aerial delivery of rabies vaccine baits to foxes (Vulpes vulpes) is less effective in hilly areas, because the density of baits per unit surface area is lower on slopes (Vuillaume et al. 1997), and aerial delivery is difficult in urban and suburban areas, which usually require delivery by hand (Müller et al. 2005).

Where wild mammals are organised into spatially distinct but inter-connected populations, the concept of metapopulation dynamics can be useful for predicting the likely impact of management interventions. Mathematical models to investigate optimal immunisation strategies, for example, suggest that for comparable levels of disease control, fewer individuals within a population are required to be vaccinated if they exist within metapopulations, than in a homogenous population of the same size (May and Anderson 1984). The local vaccination threshold necessary to eradicate a disease may be highest among high-density populations that are poorly connected, where individuals that are in contact with a given individual are not in contact with each other (Keeling 1999).

\subsubsection{Habitat Quality and Seasonality}

Landscapes can be dynamic structures, owing to seasonal changes in climate and vegetation growth. Food availability in particular may strongly influence intra- and inter-specific patterns of contact amongst mammals, with consequences for hostpathogen dynamics. For example, the seasonal availability of fruit may be associated with enhanced abundance and aggregation of mammals. This may help explain the seasonally increased incidence of Ebola haemorrhagic fever among Western gorillas (Gorilla gorilla) and common chimpanzees (Pan.troglodytes), which congregate in 
areas of high fruit abundarice (Pinzon et al. 2004). As seasonal changes in weather patterns are relatively predictable they may help improve the targeting of prophylactic campaigns or changes to management practices. For example, since uptake of vaccine baits by red foxes is higher during the summer, vaccination campaigns against rabies using oral baits are more successful when undertaken at this time of year (Flegglin.et al. 2004). Such variations in bait uptake may relate to seasonal differences in the behaviour of the target species or the availability of alternative food sources.

\subsubsection{Habitat Corridors}

The preservation and creation of corridors of favourable habitat have been widely used by conservationists to provide connections between isolated habitat patches, and so promote the persistence of endangered species through increased genetic transfer between otherwise discrete populations. However, a downside to enhanced connectivity is that it may promote the persistence and spread of diseases between populations. Habitat corridors may allow disease to persist in metapopulations where it would have otherwise gone extinct by virtue of low host density. Occasional movements of infected individuals between metapopulations connected by corridors can result in the transportation of pathogens and potentially the re-seeding of infection. Indeed, persistence of classical swine fever (CSF) in wild boar (Sus scrofa), is more likely in populations comprised of a high number of connected metapopulations, and if these connections are defined by the presence of habitat corricors (see Box 8.2).

Box 8.2 The dynamics of classical swne fever in wild boar

Across much of Europe, wild boar (Sis scrofci) are considered to be areserroir of classical swine fever (CSF); an infection that causes significanteconomic losses to the pig farming industry (Laddomada 2000 Moennig 2000 Artois et al 2002). Animal health authorities ate therefore interested in deter mining the factors that may play a tole:in the spread and persistence of $C S F$ in wild boar populations (Artois et:al. 2002). Environmental factors in particular, may influence the probability of contacts between social groups of boar. These include the continuity of forested habitat and the local density of wild boar; which is related to both food availability and hunting pressure (Rossi et al: 2005a).

CSF spreads as a continuous wave between contiguous administrative regions in Europe. This suggests that virus spread is more dependent on local. contacts between boar than on long distance dispersal (Rossi et al. 20.05b). and is consistent with their relatively sedentary habits. As wild boar movement patterns largely reflect the distribution of the forested habitat that provides them with food and shelter, so. CSF transmission is determined by: 
Box 8.2 (contimued)

forest continuity. At the scale of an epizootic, in smaller, isolated forests the emergence of .CSF is delayed and disease prevalence is lower compared to larger wooded areas. However, the relationship is more complex, because the effects of forest continuity (connectivity) and local boar density interact. Consequently, in small forested areas low wild boar density decreases the probability of CSF emergence and disease intensity (threshold effect), but within continuously forested areas (green corridors) CSF spreads regardless of boar density. In this environment, only significant barriers to boar movement, such as large rivers and fenced highways, may prevent disease spread (Laddomada 2000; Rossi et al. 2005b).

Environmenta] factors may also affect disease persistence after CSF has emerged and spread. CSF does not seem to persist locally, but it will remain in large forested areas where local epizootics are not in phase and cyclically recolonises uninfected patches (metapopulations) of wild boar (Rossi et al. 2005a; Rossi etal. 2005b). Within a large, connected landscape, virus persistence is not homogeneous, but occurs mainily in regions where wild boar density is thigh (Rossi et al. 2005a). This suggests that within a connected Iandscape, local areas of thoh boar density enhance the probability of a chain of transmission and this is possibly related to high local food availability and birth rates (Fig. 8.1). The combined effects of forest continuity and local density result tha strong courelation betweenthe persistence of CSF andwild thoar pepulation size (FIt.8.2)

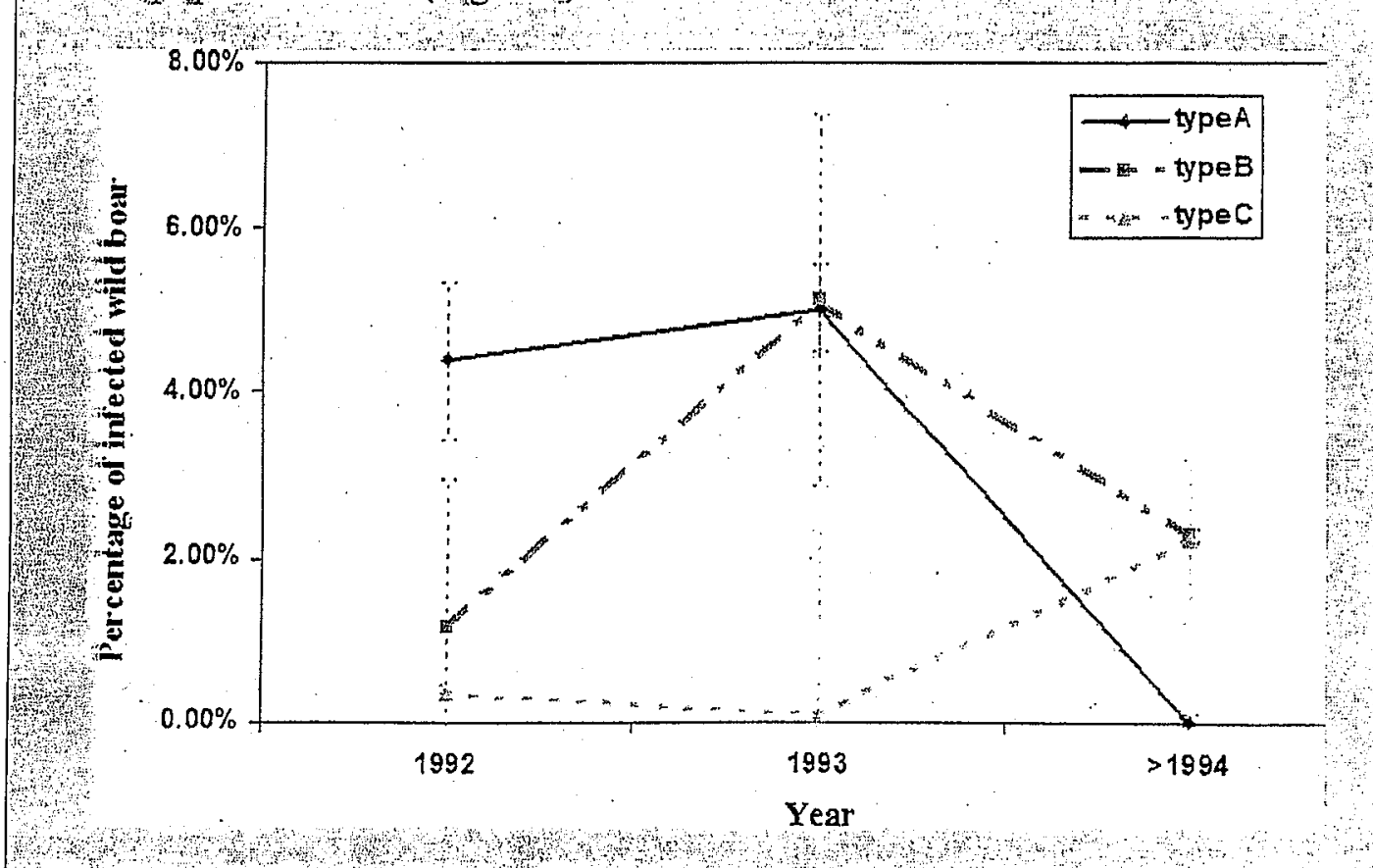

Fig. 8.1 From 1992 to 1997 intthe Vosges mountains (France) the percentage of infected wild boar depended on the continutity of the forested habitat (green conridors): the peak of infection was delayed and lower in townshps located in small and isolated forests (type C), compared to those located in large forests (types A and B) (Rossi et al 2005a) 


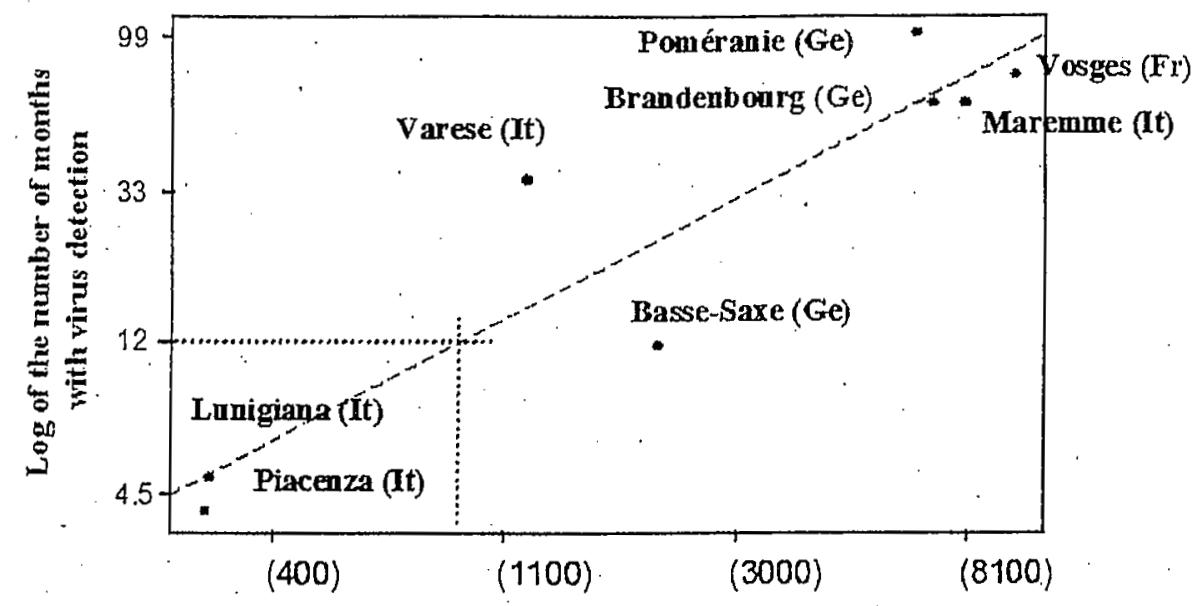

log of hunting bags

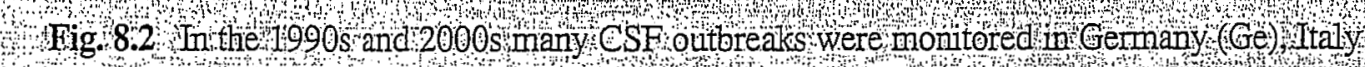

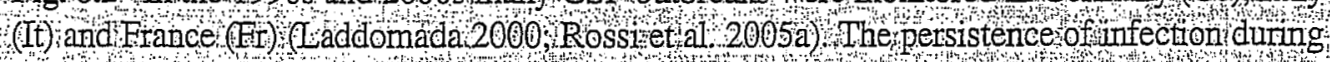

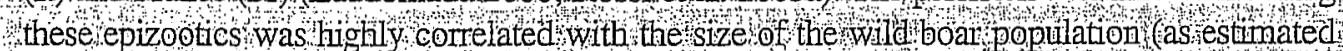
rfonthunting bags:

The nature of connections between patches strongly influences disease spread and persistence in wild populations. For example, CSF among wild boar, and rabies in red foxes, spreads along forested corridors (Real and Childs 2005; Rossi et al. 2005a), whereas rabies in raccoons (Procyon lotor) is dispersed across unforested areas (Smith et al: 2002). The identification of such relationships can allow predictions to be made about the likely course of disease spread. However, corriclors may not be as obvious as strips of woodland, particularly among more mobile species, and long-distance seasonal migrations may provide opportunities for the translocation of disease between distant regions along ill-defined corridors. Nevertheless, if data are available on migratory routes, then useful predictions of disease spread may be possible. Acquiring such information is likely to be much easier for long distance migrations of terrestrial rather than marine mammals.

\subsubsection{Barriers}

Managing disease at the local scale may influence overall transmission rates but inght not necessarily.lead to the desired level of disease control. Therefore, the area over which disease control is to be exerted must be clearly defined, and barriers, corridors and migratory routes must be taken into account. Täeally, this area should include all connected suitable habitat and population patches, but in reality these may be difficult to define, or too large to encompass (e.g. habitat patches at either 
end of a long-distance migration route). In France, during an outbreak of CSF originating in wild boar in the Vosges Forest, the putative infected area was defined using motorways and rivers that would probably limit disease spread by providing barriers to wild boat movement (Rossi et al. 2005b). The same approach was used to delineate areas within which Eurasian badgers (Meles meles) were culled as part of a study of the effects of. wildlife management on bovine tuberculosis (bTB) in cattle in Ireland (Griffin et al. 2005). In such instances the choice of 'barrier' is critical, and must be based on a clear understanding of which features in a landscape will impede animal movements.

The presence of barriers (e.g. rivers, roads, lakes) is particularly relevant for disease management planning because they may slow down or prevent the spread of some diseases amongst wild populations. For example, reduced contiguity among social group territories is predicted to be associated with reduced bTB prevalence among Eurasian badgers (Wilkinson et a]. 2004). Landscape features that may inhibit the spread of raccoon rabies in the USA have been identified by fitting observed data to mathematical models. Large rivers were associated with a seven-fold decrease in the local rate of transmission among habitat patches containing raccoons, and together with long-distance translocations were sufficient to explain the spatial pattern of rabies progression in Connecticut (Smith et al. 2002). This approach also successfully predicted the dynamics of rabies invasion in New York State (Russell et al. 2004).

For disease management purposes, it is important to note that while barriers may prevent disease spread between discrete populations, they may exacerbate the problem within the infected population along the barrier interface (Smith et al. 2002). Moreover, if used to aid disease prevention, by for example vaccination, then barriers must be sufficient to restrict emigration from the treated population. This is necessary because if host density increases in the vaccinated area due to the absence of disease, it could encourage dispersal of individuals (both vaccinated and unvaccinated) into the surrounding unvaccinated populations, thereby allowing disease to persist in the peripheral areas.

The configuration of suitable habitat patches and barriers may also affect the logistics and likely success of management efforts, because they influence the distribution and local density of hosts and the pattern of contacts between metapopulations. Mathematical modelling was used to predict the efficacy of culling brushtail possums (Trichosurus vulpecula) to control bTB under different scenarios of metapopulation patch arrangement (Fulford et al. 2002). The results showed that when patches of possum habitat were distributed as a chain (e.g. riparian habitat) or a loop (e.g. a woodland surrounding a lake), the model predicted that it was necessary to cull in several linked patches in order to counteract migration and thus eradicate the disease. The importance of curtailing immigration was further illustrated by the observation that when targeting control at a single patch surrounded by other patches to which it was connected, eradication was theoretically possible only if an exceptionally high culling rate was employed. Strategies to reduce the impact of immigration and so improve disease control were predicted to include culling in either the surrounding habitat patches only, across all patches, or in a single patch and a surrounding buffer zone designed to sever migration routes. 


\subsubsection{Scale and Clustering}

Clustering of pathogens in the environment can lead to hotspots of disease at local, regional, national and international scales. Infection with Mycobacterium avium subspecies paratuberculosis (the causative agent of Johne's disease in cattle) clusters in some populations of European rabbits (Oryctolagus cuniculus). Infection is clustered locally in rabbits within regional hotspots in Scotland (Judge et al. 2005b). Rabbit distribution is also clustered at national, regional and local scales, being influenced by availability of suitable habitat patches and the structure and quality of corridors between them (Wilson et al. 2002; Carvallo and Gomes 2003). Such clustering of disease may allow effective targeting of management efforts at the host species if hotspots are geographically stable, although this approach may not be without its problems (see Chapter 7) and its success relies crucially on the accurate identification of the hotspots. This requires the collation of suitable data on disease incidence or prevalence in the target host, or a proxy for this. such as levels of infection in sentinel species. In order to optimise disease control efforts, it may also be necessary to determine the distribution of infection within the hotspots themselves.

The scale at which disease is studied can have a considerable effect on the subsequent impression of its spatial and temporal distribution. Taking a 'snapshot' at a particular spatial or temporal scale can lead to serious misrepresentation of the disease status of an area, thus risking misinforming any management programme. If hotspots are not stable in space and time then subsequent targeting of hosts within discrete patches may, at best, be ineffective. In this case it may be more profitable to target comidors through which pathogens (and/or their hosts) may spread, in order to break the transmission chain.

\subsection{Targeting Pathogens and Vectors}

The most obvious direct method of targeting pathogens in the environment is by disinfection. Chemical disinfection of drinking water has been widely practiced to control anthrax in wild game mammals in southern Africa, but is not appropriate in many circumstances, such as in large water bodies (Berry 1993). This method is only likely to be successful where localised foci of pathogens can be identified, since wider scale disinfection of the environment is likely to be uneconomical, and potentially environmentally damaging.

The carcasses of infected animals may represent highly localised foci of infection. Trichinella spiralis (the causative agent of trichinosis), for example, is transmitted during scavenging. Also, the investigation of infected wildlife carcasses by brushtail possums, red deer (Cervus elaphus) and domestic cattle, particularly after they have been opened up by scavengers, is considered to be the main route of interand intra-specific transmission of $M$. bovis among wild mammals in New Zealand (Nugent 2005). Carcasses also play an important role in the transmission of anthrax. 
in parts of Africa where the removal and burial or burning of wildlife carcasses has been central to efforts to control the disease in wild mammals. Although it is unlikely that all carcasses can be located, even following intensive searches, reducing the overall avaitability of such sources of infection by disposing of what can be found, may be expected to provide some benefits. Nevertheless; the effectiveness of this approach is not clear, as when employed during disease outbreaks in wild birds, it does not appear to have reduced avian mortality (Wobeser 2007).

Vectors, and the free-living stages of parasites, can be indirectly targeted by manipulating the environment to make it unfavourable for their persistence. For example, removal of vegetation from. Acacia savannalh in sub-Saharan Africa rendered the environment inhospitable to tsetse flies (Glossinc spp., the insect vector of Trypanosoma spp.), thus controlling trypanosomiasis and Chaga's disease in resident wild mammals, livestock and humans (Molyneux 1982). However, such action may not be without collateral ecological costs, and in this case the resulting habitat was also rendered unsuitable for wild mammal populations that had traditionally foraged there (Molyneux 1982).

Where pathogens persist in the environment in the faeces of infected hosts they may pose a risk of infection. $M$. bovis bacilli for example, may survive in the faeces of infected Eurasian badgers, particularly in dark, moist environments, but are vulnerable to desiccation and uitraviolet light. Badger faeces are often concentrated at latrine sites, which may represent a potential source of bTB infection for cattle. It has been suggested that introducing cattle to pasture in the afternoon would maximise the exposure of bacilli present in badger latrines to the weather, and hence reduce their infectivity to grazing livestock (Phillips et al. 2003).

Direct targeting of insect vectors with insecticides has been widely practiced in the past, but has fallen out of favour owing to the problems of insecticide resistance and health risks to humans and livestock. In recent years interest has focused on integrated approaches to vector control, which include environmental management, chemical, biological and mechanical control (Lacey and Lacey 1990). Many species of anopheline and culicine mosquitoes carry pathogens causing a variety of diseases such as malaria, Japanese encephalitis, West Nile virus and Rift Valley fever. Intermittent irrigation, flushing fields and changing the timing of crop plantings have been used to discourage mosquito breeding in rice producing areas, in order to reduce disease risks for humans and livestock (Lacey and Lacey 1990). Similar approaches might be applicable for the control of pathogen vectors for wild mammals.

\subsection{Targeting Hosts}

Direct targeting of wildlife hosts for disease management has in the past often involved the reduction of population density by culling. (see Chapter 7). Environmental manipulations may provide an alternative means of reducing intra and inter-specific contact rates, through their effects on mammal distribution and local density. However, since mammals are typically highly mobile and make complex decisions regarding space 
use and movement patterns, the outcomes of environmental manipulations targeting hosts may be less easily predicted than those directed at pathogens or vectors.

A reduction in the availability of crucial resources will result in a concomitant reduction in the abundance or distribution of a population. If environmental carrying capacity is pushed sufficiently low so as to reduce the population below the density threshold at which a pathogen can persist (i.e. where $R<1$; see Chapter 3), then infection should disappear from the population.

\subsection{Manipulating Host Density and Behaviour}

Optimal foraging theory predicts that animals will distribute themselves according to the availability and abundance of resources. Hence, higher densities of individuals are expected in resource rich patches, with lower densities in sub-optimal areas. Consequently, local density may be suppressed by reducing the availability of critical resources, such as food or shelter, or distributing them more evenly across a landscape. However, such approaches are not without their potential problems. Reductions in the availability of resources could in the short-term result in malnutrition and hence increased susceptibility to disease. Also, the dispersal of animals seeking alternative food sources could potentially spread disease if infected individuals ranged further and made contact with susceptible hosts elsewhere. Finally, the use of environmental manipulation to reduce food resources may cause significant suffering (starvation), particularly among more sedentary species, and therefore raises concerns over whether such an approach is ethically acceptable.

In each situation the resource requirements and likely behavioural responses of wild populations need to be understood in some detail before environmental manipulation can be seriously considered as a disease management tool. Responses of host populations may be complex and can defy simplistic assumptions. For example, the population density of red foxes in temperate Eurasia and North America influences the spread and incidence of rabies. As fox distribution and density are dependent on the availability of food and shelter, it seems reasonable to expect that fox density could be influenced by manipulating the distribution and abundance of these critical resources. In practice however, because foxes are highly adaptable and can exploit a diversity of food items and environments, attempts to control rabies outbreaks through environmental manipulation (Steck 1982) have met with far less success than culling (Müller 1971) and vaccination (Holmala and Kauhala 2006). This is likely to be the case for other adaptable, generalist species with broad diets and habitat requirements.

The local density of wild mammals has been profoundly altered by changing agricultural practices (Cimino and Lovari 2003), burning (Van Dyke and Darragh 2007); and planting unpalatable foods (Conover 1991). Attempts to alter the density of wild mammal populations by manipulating resources, whether for the purposes of pest control, game production or conservation may also have consequences for disease dynamics. For example, diversionary feeding strategies have been employed 
in order to discourage wildlife from congregating in sensitive areas where they were considered to cause damage or nuisance, and supplementary feeding has been widely employed for game production. In the context of disease control however, supplementary feeding areas can themselves pose a risk of enhanced transmission by encouraging aggregations of individuals. Large numbers of white-tailed deer (Odocoileus virginianus'), congregated at supplementary feeding stations in Michigan, USA, and the local increases in deer density were implicated in an increased prevalence of bTB amongst wild deer and domestic cattle herds (Miller et al. 2003). Deer culling was successfully employed to reduce local deer densities below the threshold at which bTB could persist. However, restrictions on the supplementary feeding of deer also made a major contribution to the reduced prevalence of bTB in both deer and cattle (Miller et al. 2003). The dispersed planting of attractive food sources across the landscape may provide an alternative means of reducing local densities of herbivores.

Predator control is usually implemented with the intention of protecting prey populations that are of economic or conservation value. But the actions of predators may influence levels of disease in prey populations, by for example removing heavily infected individuals and reducing prey density. For some density-dependent diseases, predator removal has the potential to increase disease incidence within the prey population by allowing their local density to increase. The converse may also be true, such that an improvement in resources for predators may increase their abundance or predation success rate, and thereby disperse or reduce the density of their prey, and so potentially impede disease spread. However, unless predators are maintained at artificially high levels it is likely that the density-dependent feedback of a reduced or dispersed prey population will lead to a reduction in predator abundance in time, thus providing only short-term disease control until an equilibrium is reached between predators and their prey. An alternative scenario is that a high density of predators may promote high local abundance of pathogens that may be transmissible to other animals sharing the same environment. These hypotheses have yet to be tested empirically, and other outcomes are possible, so we are at a early stage in understanding how the manipulation of predator pressure could be used as a tool to control disease in prey populations. Nevertheless, the potential role of predator populations should be considered when developing any plan to manage disease in a wild mammal population.

\subsubsection{Disease Spread}

It is possible that the rate at which disease progresses within a population may influence the extent to which it can be controlled through environmental manipulation. The differing potential effects of habitat heterogeneity on disease spread were identified in a model simulating a chronic (i.e. bTB) and an acute (i.e. rabies) infection in Eurasian badgers. The model outputs suggested that increasing habitat heterogeneity would lead to a gradual decrease in bTB prevalence. However, a 
threshold effect was detected for rabies transmission, such that low levels of habitat heterogeneity had no effect on transmission, but high levels limited its spread (Smith and Wilkinson 2002; Wilkinson et al. 2004). These effects probably arose as a result of the different ways in which chronic and acute diseases persist and spread across landscapes. A chronic clisease, such as bTB, does not require a high frequency of host contacts in order to persist since infected individuals can survive over longer timescales. Hence, increasing habitat heterogeneity should be expected to maintain chronic diseases in localised foci, which should fade with time in the absence of host contacts. In contrast, an acute disease, such as rabies, requires a higher frequency of host contacts in order to persist and so also requires a minimum level of habitat connectivity to ensure sufficient host interactions. The implication - is that enhancing habitat heterogeneity may in some cases be used to manage disease spread in wild mammal populations by controlling contact rates, and the benefits may accrue quickly, but in the case of a rapidly progressive disease this is only possible after a contact rate threshold has been reached. For a slower progressing disease, the benefits may not accrue so quickly. At the moment these are only theoretical possibilities as no empirical evidence has yet been generated experimentally.

\subsubsection{Reducing Susceptibility to Disease}

Nutrition influences immune system functioning and hence susceptibility to disease (Lochmillar and Deerenberg 2000; Wobeser 2006). The availability of essential nutrients; protein and energy are directly associated with habitat quality and can be influenced by numerous factors. Density-dependent competition may decrease the ability of some individuals to acquire sufficient food resources, reducing their overall protein and energy intake. The competition between conspecifics that may arise as population density increases is also likely to cause stress; which can impact adversely on the performance of the immune system. It follows that reductions in population density, below the level at which inter-specific competition for resources is detrimental, could potentially improve the physical condition and resilience of individuals to disease: However, accurately predicting when this point has been reached is a considerable challenge. In addition, the demographic and behavioural consequences of reducing host population density may be counter-productive for disease control for other reasons, (see Chapter 7).

The absence of adequate shelter for the purposes of thermoregulation, predator avoidance and rearing young is likely to be another potentially important cause of enhanced stress. Therefore, management of the environment in ways that maximise the availability of suitable cover may help to decrease stress and disease susceptibility among some mammals, although of course this may also increase host density.

As disease susceptibility can vary between conspecifics of differing sex and age classes (see Chapter 2), the effects of habitat quality on disease occurrence may exhibit similar variation. Such potential differences will need to be considered when planning disease management through environmental manipulation. 


\subsubsection{Reducing Transmission Between Wild Mammals and Livestock}

Transmission of pathogens at the wilclife-livestock interface cem occur in both directions and may therefore pose a threat to either agriculture or conservation. Foot and mouth disease (FMD) in domestic cattle serves as a case in point, because although they are the most important source of infection for wild mammals on many continents, in parts of Africa they are themselves susceptible to transmission from a reservoir of infection in wild buffajo (Symcerus caffer) (Bengis et al. 2002).

The most obvious means to prevent contact between wild and domestic mammals is the use of fencing. Numerous fence designs have been successfully employed to this end (Vercauteren et al. 2006) but the cost and practicality of fencing extensive areas may limit the range of potential applications. Moreover, fences may be ineffective if not deployed at a sufficiently large scale or if positioned far from the disease front. For example, inadequately positioned fences failed to prevent transmission of brucellosis between bison (Bison bison) and cattle in the USA (Cheville et al. 1998). Numerous national parks have constructed high fences either to contain wild mammal populations or to prevent access from those outside (Kassilly 2002; Whitehouse and Kerley 2002; Sievers 2004; Walter et al: 2005), and they routinely deploy significant resources for their periodic inspection and repair. Typical problems include damage from water run-off, bad weather, fallen trees and vandalism. Electric fences have been designed specifically for the purposes of restricting the movements of wild manmals and have been deployed in South Africa and Zimbabwe in order to protect cattle from bTB and FMD transmission from wild mammals (Taylor and Martin 1987). More recently 'invisible fences' have been tested to assess their efficacy at reducing contact between livestock and wild mammals (see Box 8.3).

$4+2$ m

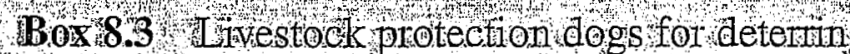

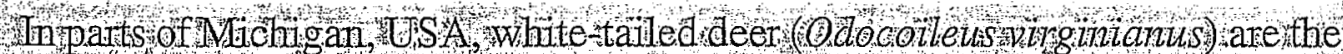

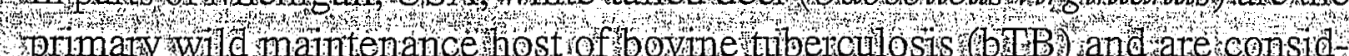

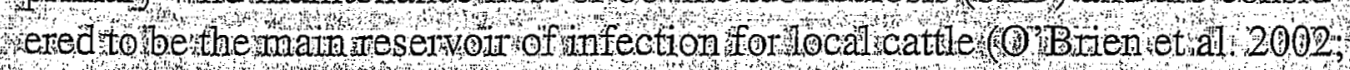

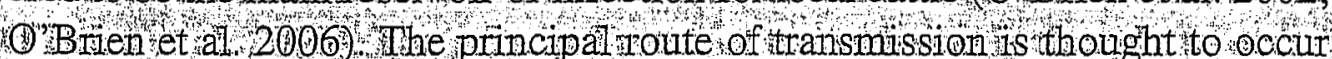
When deer contaminate cattle feed put out on pasture, (Palmer et al 2001 , (o)Brien et al. 2006) al tholghadirect contactcannot be discounted. Methods to reduce deer activity near cattle may offer options to control diuect andindirect disease transmission, but while field-scale exclusion may be effective, deerproof fencing may not always be appropriate.

For thousands of years, livestock producers have used domestic dogs to deter predators from pastures and paddocks. It follows that dogs could potentially be used to reduce dureat and indirect contact between white-tailed deer and cattle 
and hence contribute to disease control. In a field trial of this approach, dogs. were kept alongside cattle within discrete areas of pasture on ateer farm where they could be surrounded by an artificially high density of cleer. Dogs were kept within the enclosures by an Invisible Fence (IFCO Enterprises, Malvern; Pennsylvania, USA) and cattle were confined using a traditional electric fence. The Invisible Fence system involved each dog wearing a collar carrying an electronic device that responded to a signal from aw wire encircling the enclosure. The collars emitted an auditory cue when a dog approached to within 1 m of the wire and an electric shock if they failed to move away The dogs were quickly conditioned to the Invisible Fence, and treated it as if it were a phy sical boundary The results showed that dogs were effective at substantialy reducing deer incursions onto pasture and imost entirely prevented contact between deet ande either cattle or their feed, even at high deer densities.

Dogs specifically tratned to remain with grazing cattle nay therefore offer a practical tool to minmise contact be tween deer and cattle, and thereby limit opportunities for transmission of bTB and potentially other nfectious diseases. Even in larger pastures, dógs may effectively exclude deer from using spatially concentrated sources of cattle feed, which probably present the greatest risks of transmission from deer to cattle Luyestock protection dogs maystherefore proxide av valuabie biosecurity toof, particularly tor small cattle operations andithe use ofmodern invisible bativer systems mayfacilitate their efficient deployment where traditienal physical bartiers are not approptiate.

The effect of physical fencing on the behaviour of non-target species should be considered prior to installation. Fencing along waterways and highways may have delayed wolf (Canis lupus) population expansion in Spain for nearly two decades because they obstructed dispersal routes (Blanco et al. 2005). In sub-Saharan Africa fences have been used to segregate wild mammals from livestock for disease contro] (Molyneux 1982) but in Kruger National Park, South Africa they also severed a wildebeest (Connochaetes taurinus') migration route (Whyte and Joubert 1988). Fenced motorways may prevent CSF spread between wild boar populations but they also constrain lynx (Lynx lynx) dispersal (Rossi et al. 2005b; Klar et al. 2006). Restricting dispersal may also have an undesirable impact on disease management if the density of hosts inside fenced areas increases and so enhances transmission rates.

Various types of deterrent that have been employed to protect crops and other resources from wild mammals could potentially also be used to influence contact rates with domestic. stock and hence disease transmission risks. An example would be the use of domesticated animals (usually dogs) as guardians of livestock or farm facilities (see Box 8.3). Devices employing visual (e.g. scarecrows and predatormimicking devices) and auditory (e.g. exploders and distress calls) stimuli have been used as area deterrents, although these approaches may result in eventual habituation (Vercauteren et al. 2005). In general, such devices are more effective if 
they are animated (e.g. by using automated motion sensors), and if the stimuli are unpredictable and associated with a strong negative experience. The use of deterrents is likely to be most appropriate when the aim is to deter wild mammals from a specific area, such as farm buildings or a field of livestock, where risks of disease transmission are deemed to be high.

A variety of changes to domestic animal husbandry practices may help to reduce the risks of transmission of infection from wild mammals. Livestock that are housed in facilities to which wild manmals can gain access may be exposed to direct contact or environmental contamination from infectious hosts (Dolan 1993; Flanagan 1993; Hutchings and Harris 1997; Meerburg et al. 2006; Ward et al. 2008a). Where it is practicable, exclusion of wild mammals from such locations is likely to be a worthwhile livestock biosecurity measure. However, potentially infectious excretions may also be distributed across open pastoral landscapes, where the prevention of exposure to domestic stock may be more difficult.

\subsection{Turning Information into Policy}

Increasingly, policy development in many countries is required to be evidencebased, and this provides scientists, conservationists and land or wildlife managers with opportunities to influence the opinions of policy makers. Information collected with scientific rigor can provide a robust and defensible evidence base, but the length of time it can take to collect may frustrate policy makers. Hence, it is not uncommon to find policy underpinned by observation and anecdote as a substitute for scientific evidence. However, there are considerable risks associated with sources of evidence that are not robust, and are subject to selective personal interpretation. In circumstances where environmental manipulation is being considered for disease control purposes, few empirical data may be available, but it is nevertheless important that whatever information can be obtained is assessed in a systematic and objective manner. Qualitative risk assessment (see Chapter 9) may provide a useful framework for this purpose.

An excellent example of a strategy considering the potential impacts of a wildlife disease management plan, is the environmental impact statement on the control of chronic wasting disease (CWD) in white-tailed deer populations produced by the Wisconsin Department of Natural Resources, USA (Bartelt et al. 2003). The authors reviewed what was known about the pathology, transmission and detection of CWD, deer ecology and behaviour, and how they might affect the spread of infection, how other states managed the disease and contemporary control methods. They explored options for controlling wildlife diseases (including doing nothing) and the potential consequences for a variety of stakeholders including state agencies, hunters, landowners, farmers, wildlife enthusiasts, local businesses and native American Indian communities, and potential impacts on vegetation and animal communities. The comprehensive report served to inform both decision makers and the public of the likely consequences of options to control CWD. 


\subsection{Changing Attitudes and Behaviour}

Manipulation of the environment may offer opportunities to manage disease in wild mammals without resorting to potentially controversial lethal control or costly vaccine development and deployment, and so may be an attractive option for policy makers. However, environmental management is likely to require the co-operation of several key stakeholders (e.g. farmers and other land managers) and this raises a major challenge for policy makers. These parties may be reluctant to alter their long-established management practices, especially when the benefits may be uncertain or take a long time to accrue. For example, whilst the potential risks of disease transmission from wild mammals via contamination of livestock feed had been clearly demonstrated (Hutchings and Harris 1997; Garnett et al. 2002; Daniels et al. 2003a), few UK farmers appeared willing to invest in the necessary protective. husbandry measures (Bennett and Cooke 2005). Moreover, wild mammal populations transcend land ownership boundaries, and disease management strategies may therefore require co-ordinated action amongst many parties. Achieving consensus on a disease management strategy may however be difficult, particularly where neighbouring landowners have different values and opinions. The same will be true for all other sectors of society who may have an interest in the issue, including stakeholder groups, the general public, government policy makers and politicians.

Understanding the prevailing attitudes of stakeholders and how to change them in the face of scientific evidence is a substantial challenge for the development of sustainable approaches to wildlife disease management. Hence; the discussions that follow are of generic importance, although they are particularly relevant to environmental management programmes because these often require co-ordination across. landscapes and land-ownership boundaries, and are therefore hostage to the values, attitudes and opinions of multiple stakeholders.

\subsubsection{Understanding Attitudes}

One way to enhance adoption of innovation is to understand how people make decisions. Once this process is better understood, it will become easier to influence it in order to encourage people to adopt practices related to disease management. Many farmers, for example, are unusual in that their business interests, lifestyle and culture are all closely related. As a result, their decision-making processes are influenced not only by financial considerations, but also by a range of social factors, such as the age and structure of the family, sources of off-farm income and their connection to the local community (Potter and Gasson 1988). These sociodemographic issues can easily affect farmers' attitudes to risk, willingness to invest large sums of money and their likelihood to change long-standing practices (Edwards-Jones 2006). Decisions are also likely to be influenced by people's fundannental personality; attitudes and objectives (Edwards-Jones et al. 1998; Willock 
et al. 1999). Early adoption of environmental schemes and improvements to animal health and welfare, is often linked to a farmer having a personality and set of attitudes that are open to new ideas (Austin et al. 2005; Dutton et al. 20(18). Typically, only a minority will adopt new ideas quickly, a larger number will consistently resist change, while most may adopt change over time as their.social and financial situation permits.

\subsubsection{How to Influence Attitudes and Behaviour?}

Although there is no single blueprint for bringing about behavioural change, the key elements of a successful campaign typically include:

- Communicating a convincing message

- Gaining trust with the stakeholder community

- Embracing stakeholder participation

- Developing practical demonstrations

- Developing credible champions for the message

- Minimising administrative burdens

- Removing perverse incentives

- Supporting the campaign with wide scale communication

- Helping stakeholders feel good about what they have achieved

\subsubsection{Communicating the Message for Change}

It is vital that the basic message about why change is necessary is credible and makes inherent sense to stakeholders. It is likely to be necessary to demonstrate that a management approach can deliver net:benefits to the stakeholder, before they can be expected to implement or accept such measures themselves.

While benefits may be demonstrated to scientists and policy makers through experimental investigations, land managers may be more readily convinced by practical demonstration in a realistic setting, such as a working farm. Preferably such a farm would be managed by someone who is trusted and respected (i.e. a champion). It is clearly important to have a good understanding of the financial costs and benefits of any environmental manipulation and these may be presented in the form of a series of investment appraisals if net benefits resulting from behavioural change are expected to accrue to a business. If most benefits are expected to be external to the business, such as an improvement in the health of wild animals, then it may be more difficult to make the case for change financially appealing to business stakeholders.

In order to consider the wide-scale benefits that may accrue to society from changed behaviour, economists tend to undertake cost-benefit analyses (CBA; see Chapter 5). CBA requires the identification and valuation of all elements of a system that will be impacted by some intervention. Benefits may be relatively straightforward, such as 
increased profit for local businesses, but they may well also include beneficial changes in so-called 'non-market goods' such as landscape, biodiversity and animal welfare. Although these benefits do not typically have market prices associated with them, economists use a range of techniques to estimate their monetary value (see Chapter 5). Through considering all relevant costs and benefits in this way, the viability of a project can be determined in quantifiable monetary terms. Although CBA is a powerful and widely used technique it tends to be better suited to informing major business and policy decisions, than to persuading individual farmers to adopt certain practices. This is because in essence the CBA is suggesting that if the farmer undertakes certain actions (which may cost him time and money) other people in society may reap some of the benefits (i.e. through improved wildlife heaith). This almost always raises the inevitable response from farmers that if society is getting all this benefit then why are they not paid more for delivering it? For this reason, in many cases, it may be more productive in the long run to appeal to the farmers' better nature, rather than involve them in discussions of CBAs.

\subsubsection{Regulation, Incentives and Administration}

In many countries agricultural policy and the regulatory framework are complex. In addition, a variety of different organisations are typically responsible for the various components of the system. For example, within the UK, separate agencies are responsible for payment of agricultural subsidies, agri-environment schemes, animal health, waste disposal, food processing standards, farm worker safety and planning. However, many of the activities regulated by these different agencies interact at the farm level. This type of organisational structure is not confined to the UK, and is characterised by the typical observation that changes in one activity may relate to regulations that originate from more than one agency. This can create a frustrating and complex administrative burden, which means that changes to management practices are hindered or even prevented.

\subsubsection{Peer Support and the 'Feel Good' Factor}

A successful campaign may persuade stakeholders to change their behaviour. However, if this situation is to persist, then stakeholders require support from their peers. It is difficult for any individual to maintain a behaviour when their peers disapprove of their actions. So when planning a campaign to alter stakeholdei behaviour, it is important to use the media and other sources to communicate the message to the wider community. In this way the stakeholders will find themselves living and working in a supportive community, rather than one that is unsympathetic to their activities. Finally, nothing sustains desired behaviour like positive feedback. Communicating positive messages about stakeholder activities to other stakeholders and the wider community can be a powerful tool for encouraging sustained effort (Ward et al. 2008b). 


\subsection{Conciusions}

Environmental management has been used historically to control many diseases in wild mammals. While experimental studies demonstrating efficacy are rare, some predictions can be made on the basis of what is known about the relationships between environmental structure, marnmal hosts and their pathogens. From the evidence presented here it is clear that while environmental management may be a useful tool for the control of disease in wild mammals, its success rests on a sound unclerstanding of the ecology of the host-pathogen system. Of key importance is an understanding of how pathogens persist and spread in space and time within and between populations and environments. In this respect field studies and experiments are fundamentally important in providing robust empirical data, although this process can be frustratingly protracted. Developments in geographical and mathematical modelling tools can help by providing platforms on which to construct predictive models of disease spread and control, although their.value is directly related to the quality of input data and their post hoc validation using independent data (see Chapter 4).

It is important to consider both target and non-target impacts of proposed management plans since environmental manipulations are likely to impact on other components of ecological communities, including other hurman activities. EIA may provide a useful framework for the review and assessment of the potential impact of such approaches to disease management. However, this may be a considerable challenge given that the benefits of environmental manipulations are less certain than for other disease control methods, may not accrue directly to stakeholciers expected to undertake the manipulations and may take some time to materialise. This makes it all the more important to understand stakeholders' attitudes and values in order to develop and implement sustainable policies. 